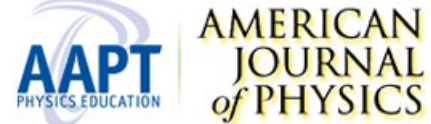

\section{Integral equations of scattering in one dimension}

Vania E. Barlette, Marcelo M. Leite, and Sadhan K. Adhikari

Citation: American Journal of Physics 69, 1010 (2001); doi: 10.1119/1.1371011

View online: http://dx.doi.org/10.1119/1.1371011

View Table of Contents: http://scitation.aip.org/content/aapt/journal/ajp/69/9?ver=pdfcov

Published by the American Association of Physics Teachers

\section{AAPT Re-register for Table of Content Alerts}

\section{Create a profile.}




\title{
Integral equations of scattering in one dimension
}

\author{
Vania E. Barlette
}

Centro Universitário Franciscano, Rua dos Andradas 1614, 97010-032 Santa Maria, RS, Brazil

Marcelo M. Leite

Departamento de Física, Instituto Tecnológico de Aeronautica, Centro Técnico Aerospacial, 12228-900 São José dos Campos, SP, Brazil

Sadhan K. Adhikaria)

Instituto de Física Teórica, Universidade Estadual Paulista, 01.405-900 São Paulo, SP, Brazil

(Received 17 August 2000; accepted 13 February 2001)

\begin{abstract}
A self-contained discussion of integral equations of scattering is presented in the case of centrally symmetric potentials in one dimension, which will facilitate the understanding of more complex scattering integral equations in two and three dimensions. The present discussion illustrates in a simple fashion the concept of partial-wave decomposition, Green's function, Lippmann-Schwinger integral equations of scattering for wave function and transition operator, optical theorem, and unitarity relation. We illustrate the present approach with a Dirac delta potential. (C) 2001 American
\end{abstract} Association of Physics Teachers.

[DOI: $10.1119 / 1.1371011]$

\section{INTRODUCTION}

The simple problem of one-dimensional quantum scattering continues as an active line of research. ${ }^{1}$ This problem has been crucial applications in studying tunneling phenomena in a finite superlattice. ${ }^{2}$ A self-contained discussion of quantum scattering in one dimension using the Schrödinger equation has appeared in the literature. ${ }^{3-7}$ Here we present a comprehensive description of the integral equations of scattering using the Green's function technique in one dimension in close analogy with the two- ${ }^{8}$ and three-dimensional cases. ${ }^{9}$ Apart from these interests in research, the present study of one-dimensional scattering is also interesting from a pedagogical point of view. In a one-dimensional treatment one does not need special mathematical functions, such as Bessel functions, while still retaining sufficient physical complexity to illustrate many of the physical concepts, such as partialwave decomposition, Green's function, the LippmannSchwinger (LS) integral equations of scattering for wave function and the transition operator, the optical theorem, and the unitarity relation, which occur in two and three dimensions.

A self-contained discussion of two-dimensional scattering has appeared in the literature. ${ }^{8}$ There is an intrinsic difference between scattering in two and three dimensions and that in one dimension. In one dimension there are only two discrete scattering directions: forward and backward along a line. This requires special techniques in one dimension in contrast with two or three dimension where an infinity of scattering directions are permitted characterized by continuous angular variables. It is because of the above subtlety of the one-dimensional scattering problem that we present a complete discussion of the integral-equation formulation in this case. This special feature of one-dimensional scattering leads to two partial waves. For a centrally symmetric potential these two partial waves are decoupled, with even and odd parity. ${ }^{7}$

Here we present a discussion of the integral equations of scattering in one dimension in momentum and configuration space for a centrally symmetric potential $V(x)=V(-x)$. Formánek ${ }^{4}$ has discussed the importance of this symmetry in obtaining mathematical simplification. Interesting things can happen in the nonsymmetric case. ${ }^{5}$ The two partial waves are coupled in the nonsymmetric case $V(x) \neq V(-x)$ and nontrivial modifications of the standard scattering formulation are needed. ${ }^{7}$ For noncentral potentials in three dimensions (in the presence of a tensor potential) the different partial-wave components also become coupled. However, in this work we shall be limited to a discussion of the centrally symmetric case.

More specifically, we present a Green-function description of scattering in partial-wave form. Also, we derive the LS equation of scattering for the wave function and obtain a suitable transition $(t)$ matrix. The on-the-energy shell (onshell) $t$ matrix element is essentially the physical scattering amplitude. We also derive the unitarity relation for the $t$ matrix and show it to be consistent with the usual form of the optical theorem in three dimensions. ${ }^{9}$

In Sec. II we present the LS integral equations. Section III deals with the unitary relations. In Sec. IV we present an illustrative application of the formalism with the delta function potential. A brief summary is presented in Sec. V.

\section{INTEGRAL EQUATION OF SCATTERING}

In one dimension the scattering wave function $\psi_{k}^{(+)}(x)$ at position $x$ satisfies the Schrödinger equation

$$
\left[-\frac{d^{2}}{d x^{2}}+V(x)\right] \psi_{k}^{(+)}(x)=k^{2} \psi_{k}^{(+)}(x)
$$

where $V(x)$ is a centrally symmetric finite-range potential in units of $\hbar^{2} /(2 m)$ satisfying $V(x)=0, r>R$ and $V(x)$ $=V(-x)$, where $r=|x|, \quad m$ the reduced mass, $E$ $\equiv \hbar^{2} k^{2} /(2 m)$ the energy, and $k$ the wave number. The scattering wave function is not square integrable and hence does not satisfy the usual normalization condition of a bound-state wave function. However it is useful in some cases to "normalize" it according to

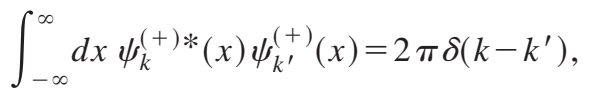


where $\delta$ is the Dirac delta function. The previous discussions on scattering in one dimension are based on the solution of the differential equation (1) for the wave function supplemented by the necessary boundary conditions. ${ }^{3-5}$ The present discussion will be based on the integral equations of scattering which we derive below.

In configuration space the Green's function $G_{0}^{ \pm}$ $\equiv \lim _{\eta \rightarrow 0}\left(k^{2}-H_{0}+i \eta\right)$, with $H_{0}$ the kinetic energy operator, satisfies

$$
\left[k^{2}+\frac{d^{2}}{d x^{2}}\right] G_{0}^{( \pm)}\left(x, x^{\prime}\right)=\delta\left(x-x^{\prime}\right)
$$

together with appropriate boundary conditions. The solution of this equation is given by ${ }^{10}$

$$
G_{0}^{( \pm)}\left(x, x^{\prime}\right)=\mp \frac{i}{2 k} \exp \left( \pm i k\left|x-x^{\prime}\right|\right) .
$$

In analogy with two and three dimensions, one can introduce the following LS equation:

$$
\psi_{k}^{( \pm)}(x)=\phi_{k}(x)+\int_{-\infty}^{\infty} G_{0}^{( \pm)}\left(x, x^{\prime}\right) V\left(x^{\prime}\right) \psi_{k}^{( \pm)}\left(x^{\prime}\right) d x^{\prime} .
$$

In operator form this equation becomes

$$
\left|\psi_{k}^{( \pm)}\right\rangle=\left|\phi_{k}\right\rangle+G_{0}^{( \pm)} V\left|\psi_{k}^{( \pm)}\right\rangle .
$$

The incident plane wave $\phi_{k}(x) \equiv\left\langle x \mid \phi_{k}\right\rangle \equiv\langle x \mid k\rangle=\exp (i k x)$ satisfies the free Schrödinger equation $H_{0} \phi_{k}=k^{2} \phi_{k}$. The \pm sign in Eq. (5) refers to the outgoing and incoming wave boundary conditions, respectively. The physical scattering problem corresponds to the positive sign.

Equation (4) when substituted into Eq. (5) leads to the following asymptotic form for the wave function: ${ }^{7}$

$$
\lim _{r \rightarrow \infty} \psi_{k}^{( \pm)}(x) \rightarrow \exp (i k x)+\frac{i}{k} f_{k}^{( \pm)}(\epsilon) \exp ( \pm i k r),
$$

where

$$
f_{k}^{( \pm)}(\epsilon)=\mp \frac{1}{2} \int_{-\infty}^{\infty} \exp \left(\mp i \epsilon k x^{\prime}\right) V\left(x^{\prime}\right) \psi_{k}^{( \pm)}\left(x^{\prime}\right) d x^{\prime} .
$$

The quantity $f_{k}^{( \pm)}(\epsilon)$ is termed the scattering amplitude. The parameter $\epsilon$ is +1 for $x>0$ and -1 for $x<0$ in the asymptotic region. Definition (8) of the scattering amplitude ${ }^{6}$ is different from that used in Refs. 3-5:

$$
\widetilde{f}_{k}^{( \pm)}(\epsilon)=\mp \frac{i}{2 k} \int_{-\infty}^{\infty} \exp \left(\mp i \epsilon k x^{\prime}\right) V\left(x^{\prime}\right) \psi_{k}^{( \pm)}\left(x^{\prime}\right) d x^{\prime} .
$$

The essential difference between the two is in the phase factor $i$ which does not influence the cross sections. However, the present definition has the advantage of leading to an optical theorem in close analogy with that in three dimensions as we shall see in the following. The scattering amplitude of Refs. 3-5 leads to an optical theorem distinct from the one in three dimensions. The physical outgoing-wave boundary condition is given by Eq. (7) for $\psi_{k}^{(+)}(x)$. There are two discrete scattering directions in one dimension characterized by $\epsilon= \pm 1$ in the asymptotic region in contrast with the infinite possibility of scattering angles in two and three dimensions. The forward (backward) direction is given by $\epsilon$
$=1(-1)$. The discrete differential cross sections in these two directions are given by

$$
\sigma_{\epsilon}=\frac{1}{k^{2}}\left|f_{k}^{(+)}(\epsilon)\right|^{2}
$$

and the total cross section by ${ }^{3-5}$

$$
\sigma_{\mathrm{tot}}=\sum_{\epsilon} \sigma_{\epsilon}=\frac{1}{k^{2}}\left[\left|f_{k}^{(+)}(+)\right|^{2}+\left|f_{k}^{(+)}(-)\right|^{2}\right] .
$$

The discrete sum in Eq. (11) is to be compared with integrals over continuous angle variables in two and three dimensions. The usual reflection $(R)$ and transmission $(T)$ probabilities are given by $T=\left|1+i f_{k}^{(+)}(+) / k\right|^{2}$ and $R=\left|i f_{k}^{(+)}(-) / k\right|^{2}$.

Here we must comment on the dimension (unit) of cross section and scattering amplitudes. Cross section is defined to be the number of events (particles scattered) per unit time per unit incident flux. The incident flux is the number of particles incident per unit time per a section of space. The section of space has units of area and length in three and two dimensions and is dimensionless in one dimension. The corresponding incident flux is measured in units of $T^{-1} L^{-2}$, $T^{-1} L^{-1}$, and $T^{-1}$ in these dimensions, respectively, where $T$ denotes time and $L$ length. The cross section is an observable and has units of $L^{2}$ and $L$ in three ${ }^{9}$ and two ${ }^{8}$ dimensions and is dimensionless in one dimension. The present cross section (11) is dimensionless. Scattering amplitude is not a physical observable and there could be some flexibility in its definition. The present definition of the scattering amplitude leads to an optical theorem which is formally similar to the optical theorem in three dimensions. Other definitions of the scattering amplitude in terms of other units are also possible, for example the one given by Eq. (9).,

Next we introduce the on-shell matrix elements of the transition operator $t$ via the following equations:

$$
\begin{aligned}
\left\langle\epsilon k\left|t^{( \pm)}\left(k^{2}\right)\right| k\right\rangle & =-2 f_{k}^{( \pm)}(\epsilon), \\
\left\langle k^{\prime}\left|t^{( \pm)}\left(k^{2}\right)\right| k\right\rangle & =\left\langle\phi_{k^{\prime}}|V| \psi_{k}^{( \pm)}\right\rangle \\
& =\int_{-\infty}^{\infty} \exp \left(\mp i k^{\prime} x^{\prime}\right) V\left(x^{\prime}\right) \psi_{k}^{( \pm)}\left(x^{\prime}\right) d x^{\prime},
\end{aligned}
$$

with $k^{\prime}=\epsilon k$ and $\epsilon= \pm 1$, so that $k^{2}=k^{\prime 2} ; \epsilon=+1(-1)$ corresponds to the forward (backward) direction. The transition operator $t$ is taken to obey $t^{( \pm)}\left(k^{2}\right)\left|q^{\prime}\right\rangle=V\left|\psi_{q^{\prime}}^{( \pm)}\right\rangle$with $q^{\prime 2}$ $\neq k^{2}$. From Eq. (6) we find that the transition operator $t$ satisfies

$$
t^{( \pm)}\left(k^{2}\right)=V+V G_{0}^{( \pm)} t^{( \pm)}\left(k^{2}\right) .
$$

The usual spectral representation of the free Green's function is ${ }^{10}$

$$
G_{0}^{( \pm)}\left(x, x^{\prime}\right)=\frac{1}{2 \pi} \int_{-\infty}^{\infty} d p \frac{\exp (i p x) \exp \left(-i p x^{\prime}\right)}{k^{2}-p^{2} \pm i 0} .
$$

Using Eqs. (14) and (15) one obtains the following explicit LS equation for the general off-shell $t$ matrix elements $\left\langle q\left|t^{( \pm)}\left(k^{2}\right)\right| q^{\prime}\right\rangle\left(q^{2} \neq k^{2} \neq q^{\prime 2}\right)$ : 


$$
\begin{aligned}
\left\langle q\left|t^{( \pm)}\left(k^{2}\right)\right| q^{\prime}\right\rangle= & \left\langle q|V| q^{\prime}\right\rangle+\frac{1}{2 \pi} \int_{-\infty}^{\infty} d p\langle q|V| p\rangle \\
& \times \frac{1}{k^{2}-p^{2} \pm i 0}\left\langle p\left|t^{( \pm)}\left(k^{2}\right)\right| q^{\prime}\right\rangle,
\end{aligned}
$$

where

$$
\left\langle q|V| q^{\prime}\right\rangle=\int_{-\infty}^{\infty} \exp (-i q x) V(x) \exp \left(i q^{\prime} x\right) d x .
$$

Using the symmetry property of $V$, e.g., $V(x)=V(-x)$, and Eq. (17) it can be shown that

$$
\left\langle\epsilon q|V| q^{\prime}\right\rangle=2 \int_{0}^{\infty} d r V(r) \cos \left(\bar{q}^{\prime} r+\epsilon \bar{q} r\right),
$$

where $\bar{q}=|q|$. One can conveniently define the momentumspace partial-wave matrix elements of the potential by

$$
\left\langle\epsilon q|V| q^{\prime}\right\rangle=2 \sum_{L=0}^{1} \epsilon^{L}\left\langle\bar{q}\left|V_{L}\right| \bar{q}^{\prime}\right\rangle,
$$

where $V_{L}$ 's are the desired partial-wave components. Using Eqs. (18) and (19) we obtain

$$
\begin{aligned}
\left\langle\bar{q}\left|V_{0}\right| \bar{q}^{\prime}\right\rangle & =\int_{0}^{\infty} d r \cos (\bar{q} r) V(r) \cos \left(\bar{q}^{\prime} r\right) \\
& \equiv \frac{1}{2} \int_{-\infty}^{\infty} d x \cos (\bar{q} x) V(x) \cos \left(\bar{q}^{\prime} x\right), \\
\left\langle\bar{q}\left|V_{1}\right| \bar{q}^{\prime}\right\rangle & =\int_{0}^{\infty} d r \sin (\bar{q} r) V(r) \sin \left(\bar{q}^{\prime} r\right) .
\end{aligned}
$$

Defining the partial-wave elements of the $t$ matrix as in Eq. (19) via

$$
\left\langle\epsilon q\left|t^{( \pm)}\left(k^{2}\right)\right| q^{\prime}\right\rangle=2 \sum_{L=0}^{1} \epsilon^{L}\left\langle\bar{q}\left|t_{L}^{( \pm)}\left(k^{2}\right)\right| \bar{q}^{\prime}\right\rangle,
$$

one arrives at the following partial-wave projection of the LS equation (16):

$$
\begin{aligned}
\left\langle\bar{q}\left|t_{L}^{( \pm)}\left(k^{2}\right)\right| \bar{q}^{\prime}\right\rangle= & \left\langle\bar{q}\left|V_{L}\right| \bar{q}^{\prime}\right\rangle+\frac{2}{\pi} \int_{0}^{\infty} d \bar{p}\left\langle\bar{q}\left|V_{L}\right| \bar{p}\right\rangle \\
& \times \frac{1}{k^{2}-p^{2}+ \pm i 0}\left\langle\bar{p}\left|t_{L}^{( \pm)}\left(k^{2}\right)\right| \bar{q}^{\prime}\right\rangle .
\end{aligned}
$$

The integration limits in the partial-wave form extend from 0 to $\infty$ as after partial-wave projection we generate the modulus of momentum variable.

\section{UNITARITY RELATION}

In order to derive the unitarity relation we write the formal solution of the LS equation (23) as

$$
t_{L}^{( \pm)}\left(k^{2}\right)=V_{L}+V G^{( \pm)}\left(k^{2}\right) V_{L},
$$

where the full Green's function is defined by $G^{( \pm)}\left(k^{2}\right)$ $=\lim _{\eta \rightarrow 0}\left(k^{2}-H \pm i \eta\right)^{-1}$ with $H=H_{0}+V$ the full Hamiltonian. Using the complete set of eigenstates of the Hamiltonian $H$, one has the following spectral decomposition of the full Green's function:

$$
G^{( \pm)}\left(k^{2}\right)=\sum_{n} \frac{\left|\psi_{n}\right\rangle\left\langle\psi_{n}\right|}{k^{2}-e_{n}}+\frac{2}{\pi} \int_{0}^{\infty} d p \frac{\mid \psi_{p}^{(+)}\left\langle\psi_{p}^{(+)}\right|}{k^{2}-p^{2} \pm i 0},
$$

where the summation over $n$ refers to the bound states $\psi_{n}$ of energy $e_{n}$ and the integration over $p$ to the scattering states $\psi_{p}^{(+)}$with the normalization conditions $\left\langle\psi_{n} \mid \psi_{n}\right\rangle=1$ and $\left\langle\psi_{q}^{(+)} \mid \psi_{p}^{(+)}\right\rangle=2 \pi \delta(p-q)$. From Eq. (25) one realizes that when this expression is substituted in Eq. (24), the integral over the continuum states will contribute a square-root cut in the complex energy plane along the real positive energy axis, so that the $t$ matrix will possess this so-called unitarity cut. The discontinuity across this cut is given by the following difference:

$$
\begin{aligned}
& \left\langle\bar{q}\left|t_{L}^{(+)}\left(k^{2}\right)-t_{L}^{(-)}\left(k^{2}\right)\right| \bar{q}^{\prime}\right\rangle \\
& \quad=-4 i \int_{0}^{\infty} d \bar{p} \delta\left(k^{2}-p^{2}\right)\left\langle\bar{q}\left|t_{L}^{(+)}\left(p^{2}\right)\right| \bar{p}\right\rangle\left\langle\bar{p}\left|t_{L}^{(-)}\left(p^{2}\right)\right| \bar{q}^{\prime}\right\rangle
\end{aligned}
$$

or

$$
\Im\left\langle\bar{q}\left|t_{L}^{(+)}\left(k^{2}\right)\right| \bar{q}^{\prime}\right\rangle=-\frac{1}{\bar{k}}\left\langle\bar{q}\left|t_{L}^{(+)}\left(k^{2}\right)\right| \bar{k}\right\rangle\left\langle\bar{k}\left|t_{L}^{(-)}\left(k^{2}\right)\right| \bar{q}^{\prime}\right\rangle,
$$

where $\mathfrak{I}$ denotes the imaginary part. When $\bar{q}=\bar{q}^{\prime}=\bar{k}$, Eq. (27) becomes

$$
\Im\left\langle\bar{k}\left|t_{L}^{(+)}\left(k^{2}\right)\right| \bar{k}\right\rangle=-\frac{1}{\bar{k}}\left|\left\langle\bar{k}\left|t_{L}^{(+)}\left(k^{2}\right)\right| \bar{k}\right\rangle\right|^{2},
$$

which leads to the following parametrization for the on-shell $t$ matrix in terms of the phase shift $\delta_{L}$ :

$$
\left\langle\bar{k}\left|t_{L}^{(+)}\left(k^{2}\right)\right| \bar{k}\right\rangle=-\bar{k} \exp \left(i \delta_{L}\right) \sin \left(\delta_{L}\right) .
$$

From Eqs. (12), (22), and (29) we obtain the following partial-wave projection of the scattering amplitudes: ${ }^{3-5}$

$$
f_{k}^{(+)}(\epsilon)=\bar{k} \sum_{L=0}^{1} \epsilon^{L} \exp \left(i \delta_{L}\right) \sin \left(\delta_{L}\right) .
$$

The two phase shifts for both amplitudes $f_{k}^{(+)}(+)$and $f_{k}^{(+)}(-)$are the same. Now one naturally defines the partialwave amplitudes

$$
f_{L}=\bar{k} \exp \left(i \delta_{L}\right) \sin \left(\delta_{L}\right)
$$

so that the total cross section of Eq. (11) becomes ${ }^{3-5}$

$$
\sigma_{\text {tot }}=2 \sum_{L=0}^{1} \sin ^{2}\left(\delta_{L}\right)
$$

and satisfies the following optical theorem: ${ }^{7}$

$$
\sigma_{\text {tot }}=\frac{2}{\bar{k}} \Im f_{k}^{(+)}(+),
$$

in close analogy with the three-dimensional case, where $f_{k}^{(+)}(+)$is the forward scattering amplitude. The scattering amplitude (9) of Refs. 3-5 leads to the distinct optical theorem $^{5}$ 


$$
\sigma_{\text {tot }}=-2 \Re \tilde{f}_{k}^{(+)}(+),
$$

where $\mathfrak{R}$ denotes the real part.

\section{DELTA POTENTIAL}

Here we present an illustrative application of the formulation using the delta potential: $V(x)=\lambda \delta(x)$. The configuration space solution of the problem is well known. ${ }^{11}$ In the following we show that the integral-equation treatment leads to the same result. The solutions are analytically known in this simple case. From Eqs. (20) and (21) we find that $\left\langle\bar{q}\left|V_{0}\right| \bar{q}^{\prime}\right\rangle=\lambda / 2$ and $\left\langle\bar{q}\left|V_{1}\right| \bar{q}^{\prime}\right\rangle=0$. Hence we have only one partial wave $(L=0)$ in this case. In this case Eq. (23) has the trivial solution

$$
\left\langle\bar{q}\left|t_{0}^{(+)}\left(k^{2}\right)\right| \bar{q}^{\prime}\right\rangle=\frac{\lambda / 2}{1-\frac{\lambda}{\pi} \int_{0}^{\infty} \frac{d p}{k^{2}-p^{2}+i 0}}=\frac{i k \lambda / 2}{i k-\lambda / 2} .
$$

In this simple problem the $t$ matrix elements are independent of the momentum variables. From Eqs. (12), (22), and (35) we find that

$$
\frac{i}{k} f_{k}^{(+)}(\epsilon)=\frac{\lambda / 2}{i k-\lambda / 2}
$$

for both $\epsilon=+1$ and $\epsilon=-1$. Consequently, Eq. (7) becomes

$$
\begin{aligned}
\lim _{r \rightarrow \infty} \psi_{k}^{(+)}(x) & \rightarrow \exp (i k x)+\frac{\lambda / 2}{i k-\lambda / 2} \exp (i k r) \\
& \rightarrow \exp (i k x)+\frac{m v_{0}}{i \hbar p_{0}-m v_{0}} \exp (i k r),
\end{aligned}
$$

where $\lambda \equiv 2 m v_{0} / \hbar^{2}$ and $\hbar k=p_{0}$. In Eq. (38) we have restored the factors of mass $m$ and $\hbar$. Equation (38) is essentially Eq. (4-95) obtained by configuration space treatment in Ref. 11. Although, in the scattering approach, Eqs. (37) and (38) emerge as asymptotic conditions, they are exact results valid for all $x$ in this simple analytically soluble problem. From Eqs. (29) and (35) we find that the phase shift $\delta_{0}$ is defined by $\tan \delta_{0}=-\lambda /(2 k)=-m v_{0} /\left(\hbar p_{0}\right) .{ }^{11}$ The boundstate energy corresponds to the pole of the $t$ matrix (35) which happens at $k^{2}=-\lambda^{2} / 4$ or at $E=\hbar^{2} k^{2} /(2 m)$ $=-m v_{0}^{2} /\left(2 \hbar^{2}\right)-$ a result well known from configurationspace methods. ${ }^{11}$ As the $t$ matrix and the scattering amplitudes are known, all relevant quantities, such as cross sections and reflection and transmission probabilities, can now be calculated. The reflection and transmission probabilities are given by $R=\left|i f_{k}^{(+)}(-) / k\right|^{2}=\lambda^{2} /\left(\lambda^{2}+4 k^{2}\right)$ $=m v_{0}^{2} /\left(2 \hbar^{2} E+m v_{0}^{2}\right), \quad T=\left|1+i f_{k}^{(+)}(+) / k\right|^{2}=4 k^{2} /\left(\lambda^{2}\right.$ $\left.+4 k^{2}\right)=E /\left[E+m v_{0}^{2} /\left(2 \hbar^{2}\right)\right],{ }^{11}$ such that $R+T=1$, whereas the cross section is given by $\sigma_{\text {tot }}=\left|i f_{k}^{(+)}(+) / k\right|^{2}+\left|i f_{k}^{(+)}(-) / k\right|^{2}$ $=2 \lambda^{2} /\left(\lambda^{2}+4 k^{2}\right)=2 m v_{0}^{2} /\left(2 \hbar^{2} E+m v_{0}^{2}\right)$.

\section{SUMMARY}

In this paper we have generalized the standard treatments of two- and three-dimensional scattering to the case of onedimensional scattering. We have introduced the concept of scattering amplitude, partial wave, phase shift, unitarity, the Lippmann-Schwinger scattering equation for the wave function, and the $t$ matrix in close analogy with threedimensional scattering. In this case there are two partial waves: $L=0$ and 1 in contrast with two and three dimensions where there is an infinite number of partial waves. In one dimension the algebra is much simpler and mostly analytic. This makes the integral equation formulation of scattering in one dimension along with its partial-wave projection easily tractable. Consequently, difficult concepts of scattering, such as the optical theorem and unitarity, are easily understandable. Hence the present formulation will be helpful for teaching the formal theory of scattering in two and three dimensions.

\section{ACKNOWLEDGMENTS}

The work is supported in part by the Conselho Nacional de Desenvolvimento-Científico e Tecnológico, Fundação de Amparo à Pesquisa do Estado de São Paulo, and Financiadora de Estudos e Projetos of Brazil.

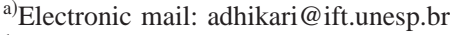

${ }^{1}$ T. Miyazawa, "Generalized low-energy expansion formula for Green's function of the Fokker-Planck equation," J. Phys. A 33, 191-225 (2000); M. Visser, "Some general bounds for one-dimensional scattering," Phys. Rev. A 59, 427-438 (1999); L. V. Chebotarev and A. Tchebotareva, "Flat resonances in one-dimensional quantum scattering," J. Phys. A 29, 72597277 (1996); M. G. Rozman, P. Reineker, and R. Tehver, "Scattering by locally periodic one-dimensional potentials," Phys. Lett. A 187, 127-131 (1994).

${ }^{2}$ R. Tsu and L. Esaki, "Tunneling in a finite superlattice," Appl. Phys. Lett. 22, 562-564 (1973); L. Esaki, "A birds-eye-view on the evolution of semiconductor superlattices and quantum wells," IEEE J. Quantum Electron. QE-22, 1611-1624 (1986); A. P. Silin, "Semiconductor superlattices," Usp. Fiz. Nauk 147, 485-521 (1985).

${ }^{3}$ J. H. Eberly, "Quantum scattering theory in one dimension," Am. J. Phys. 33, 771-773 (1965)

${ }^{4}$ J. Formánek, "On phase shift analysis of one-dimensional scattering," Am. J. Phys. 44, 778-779 (1976)

${ }^{5}$ A. N. Kamal, "On the scattering theory in one dimension," Am. J. Phys. 52, 46-49 (1984).

${ }^{6}$ V. E. Barlette, M. M. Leite, and S. K. Adhikari, "Quantum scattering in one dimension,” Eur. J. Phys. 21, 435-440 (2000)

${ }^{7}$ Y. Nogami and C. K. Ross, "Scattering from a nonsymmetric potential in one dimension as a coupled-channel problem," Am. J. Phys. 64, 923-928 (1996).

${ }^{8}$ S. K. Adhikari, "Quantum scattering in two dimensions," Am. J. Phys. 54, 362-367 (1986); S. K. Adhikari, W. G. Gibson, and T.-K. Lim, "Effective range theory in two dimensions," J. Chem. Phys. 85, 5580-5583 (1986). ${ }^{9}$ S. K. Adhikari, Variational Principles and the Numerical Solution of Scattering Problems (Wiley, New York, 1998); S. K. Adhikari and K. L. Kowalski, Dynamical Collision Theory and its Applications (Academic, San Diego, 1991).

${ }^{10} \mathrm{P}$. M. Morse and H. Feshbach, Methods of Theoretical Physics (McGrawHill, New York, 1953), 811 pp.

${ }^{11}$ G. Baym, Lectures on Quantum Mechanics (Benjamin/Cummings, London, 1981), pp. 113-116.
} 